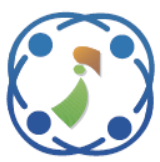

\title{
Co-Operative Directional Routing Protocol for MANET
}

\author{
Sadanand Ramchandrarao Inamdar $^{1^{*}} \quad$ SathishBabu Basavaiah $^{2} \quad$ Ravi Malleshappa Yadahalli $^{3}$

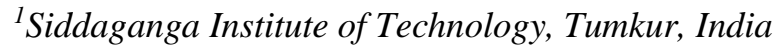 \\ ${ }^{2}$ Rashtreeya Vidyalaya Engineering College, Bengaluru, India \\ ${ }^{3}$ Shivappa Gurubasappa Balekundri Institute of Technology, Belgaum, Karnataka, India \\ *Corresponding author’s Email: sadanandrinamdar@gmail.com
}

\begin{abstract}
In Mobile Ad Hoc Network (MANET), the existing routing protocol may result in overhead and increased collisions during data transmission. In order to overcome this issue, in this paper, we propose to design a cooperation based directional routing protocol in MANET. Initially the source nodes discover neighbors by constructing the Directional Neighbour Table (DNT). Then the node with minimum transfer time is selected as best relay node. The transfer time is estimated using bio-inspired algorithm. Once the relay nodes are selected, the source node follows the Adaptive Directional Monitoring MAC (ADMMAC) protocol to transmit the data to the destination. In this technique, the nodes use the co-operative transmission mode when it requires transmitting the data packet faster. By simulation results, we show that the proposed technique minimizes the overhead and enhances the efficiency.
\end{abstract}

Keywords: MANET, DNT, Traffic, Data packet, ADM.

\section{Introduction}

Mobile Ad Hoc network (MANET) is a multihop mobile wireless network which does not have any preexisting network infrastructure or centralized administration. Due to its convenience of mobile communication, MANET is explored for numerous applications, such as network extension, ubiquitous computing, urban sensing and vehicular networking. In multi-hop wireless ad-hoc networks, designing energy-efficient routing protocols is critical since nodes have very limited energy, computing power and communication capabilities [1].

In route discovery, route to a destination will be discovered by broadcasting the query. Frequent route discovery and in some instances, additional periodic updates will cause more bandwidth being utilized and thus more energy wastage [2]. A directional antenna has certain preferred transmission and reception directions, that is, transmits or receives more energy in one direction compared to the other [3].
Use of directional antennas in MANETs creates new types of hidden terminal problems and node deafness. Deafness is defined as the phenomenon when a node $\mathrm{X}$ is unable to communicate with node $\mathrm{Y}$, as $\mathrm{Y}$ is presently beam formed in a different direction. In such an event, $X$ perceives $Y$ to have moved out of its range, thereby signaling its routing layer to take actions hence affecting the network throughput $[4,5]$.

Routing protocols including Dynamic Source Routing (DSR) [6], Ad-hoc On-demand Distance Vector (AODV) [7], Zone Routing Protocol (ZRP), Location Aided Routing (LAR) [8], and so on, use variants of a network-wide broadcasting to establish and maintain routes. Ultimately, these protocols use simple flooding for broadcasting. Simple flooding causes redundancy and increases the level of contention and collision in a network.

\subsection{Cross-Layer interaction in MANET}

Cross layer interaction is used for data transmission by sharing information among several layers such as MAC layer, network layer, transport 
layer etc. Shared database or table is created to enable layers to share information even though each layer is performing different functions. Two interfaces are created between two layers enabling bidirectional flow of information.

Directional routing protocol (DRP) [9] has provided high overhead and low efficiency. In this paper, we propose a Co-operative Directional Routing Protocol (CDRP) in MANET. Using directional neighbor table (DNT), the source node identifies its neighbors. Then, a node with minimum transfer time is selected as best relay node. After the selection of relay nodes, the source node transmits the data to the destination by following the Adaptive Directional Monitoring MAC (ADMMAC) protocol. The main objective of proposed CDRP is to minimize the overhead and enhances the efficiency rather than existing DRP [9].

Rest of this paper is organized as follows. Section 2 relates our work with the previous works. Our proposed cooperative directional routing is described in section 3. Results of our proposed approach are discussed in section 4. This paper is concluded with section 5 .

\section{Related works}

S. Chaudhari et al. [10] have proposed central authority based resource prediction mechanism considering mobility (CARPM) that predicts the resources using agents through the resource prediction agency consisting of one static agent, one cognitive agent and two mobile agents. Agents predict the traffic, mobility, buffer space, energy, and bandwidth effectively that is necessary for efficient resource allocation to support real-time communications.

S. Gonzalez et al. [11] have evaluated the scalable video streaming (SVC) over MANET by two schemes. In the first scheme, video is transmitted by means of maintaining a constant transmission rate and sending the information of all layers. The other scheme incorporates an adaptive model in which the source of traffic eliminates layers from SVC stream in order to adapt with bandwidth.

C. Lal et al. [12] have addressed the aforementioned limitations in existing quality of service (QoS)-aware routing, and the multi constraint Quality of Experience (QoE) centric routing technique for efficient transmission of multimedia traffic in MANETs. It uses large scale emulation setup, human-in-loop and hardware-inloop, QoE evaluation and real-time video transmission using multimedia software for reactive MANET routing protocol.

Y. Bao et al. [13] have improved the QoE in terms of network media transmission service, and QoE evaluation basis for adjusting the transmission control mechanism. Therefore, a kind of QoE collaborative evaluation method based on fuzzy clustering heuristic algorithm is used, which is concentrated on service score calculation at the server side.

N. Boddu et al. [14] have proposed fault tolerant multipath routing protocol simulated to study its performance. To reduce the packet loss due to route breakage, a route discovery mechanism has been used. The nodes compute multiple disjoint routes with more battery power and residual energy, to every active destination.

B. Rajkumar et al. [15] have proposed secure light weight encryption protocol for MANET, where an algorithm for providing availability with DoS resilience is used to avoid flooding packets in the network and passes other packets and also an authentication code and hash function also generated.

\section{Proposed solution}

\subsection{Overview}

In this paper, we propose to design a CDRP in MANET. Initially the source nodes discover neighbors by constructing the DNT. Then the node with minimum transfer time is selected as best relay node. The transfer time is estimated from the Network Density (ND) and Traffic Intensity (TI) information collected by ant agents. Once the relay nodes are selected, the source node follows the ADMMAC protocol to transmit the data to the destination. In this technique, the nodes use the cooperative transmission mode when it requires transmitting the data packet faster.

\subsection{Antenna model}

We consider the flat-top directional antenna model with fixed beam width $(\mu)$. This model supports multiple data rate.

The antenna sector count $\beta$ in the flat-top directional antenna model is estimated using the following Eq. (1),

$$
\beta=\frac{2 \pi}{\mu}
$$

The antenna gain, 


$$
A G(\mu)=\left\{\begin{array}{c}
1, \quad|\mu| \leq \text { beam_width } \\
0, \text { otherwise }
\end{array}\right.
$$

This reveals that the antenna holds unity gain within beam width and zero gain outside.

If gain is $A G(\mu)$, then the gain is $A G(\mu) / X$ for one of the omnidirectional antenna sectors as the transmission power is decreased by $X$.

$$
A G(\mu)=A G_{d} \approx X A G_{0}
$$

Where, $A G_{d}$ is directional antenna gain, $A G_{0}$ is sector gain of the Omni directional antenna. The range of antenna $(Z)$ is computed using following Eq. (4) based on Friis transmission:

$$
Z=\left(\frac{P_{t x} A G_{t x} A G_{r x}}{\varepsilon P_{r x}}\right)^{1 / \psi}
$$

Where, $P_{t x}$ is transmission power, $P_{r x}$ is received power, $A G_{t x}$ is transmitted gain, $A G_{r x}$ is received gain, $\varepsilon$ is constant and $\psi$ is path loss index.

\subsection{Cross-layer interactions of DRP}

DRP is an on-demand routing protocol that couples the routing layer with the MAC layer. This protocol assumes that there is a cross-layer interaction between some of the modules. In this protocol, the routing information to various destinations is stored in the Directional Routing Table (DRT) in routing layer. The DNT is shared with MAC layer. DSR maintains only the index of the node ID in a forwarding path, whereas DRP maintains node indices and the beam IDs used by the nodes to receive a packet in the forwarding path. The beam ID stored in the DRT helps the source node to estimate the angular position of its destination relative to itself. DRP uses the beam ID kept in the DRT to perform an efficient route recovery.

\subsection{Relay node selection}

The transfer time of the node can be estimated based on ND and TI information which are collected using Ant Colony Optimization (ACO) technique. This process involves two ant agents' namely Forward Ant (FANT) and Backward Ant (BANT).

Let $S$ and $D$ be the source and destination respectively.

Let $N_{i}$ be the $i^{\text {th }}$ intermediate node.

Let $E_{i}$ be the initial energy of the $i^{\text {th }}$ node.

1. Initially when the nodes are deployed in the network, FANT is generated at $S$ and routes the route set $G^{F A N T}$ is established between $S$ to $D$.

$$
G^{F A N T}=\left\{G_{s, d}^{F A N T} \mid \forall s \in \in S, \forall d \in D\right\}
$$

2. From the established route, the Network Adjacency Matrix (NAM) and node neighbour set $N_{i}$ is estimated. We can compute the node degree from NAM.

3. Each FANT selects the next hop node as per the following traffic arrival probability which is defined using state transition rule.

$$
\left\{\begin{array}{c}
\max \left\{\left[\varsigma_{i, j}(t)\right]^{a 1}\left[h_{i, j}(t)\right]^{a 2}\right\} ; w \leq w_{0}, n \in N_{i} \\
\frac{\left[s_{i, j}(t)\right]^{a 1}\left[h_{i, j}(t)\right]^{a 2}}{\sum_{x \in N_{i}}\left[s_{i, j}(t)\right]^{a 1}\left[h_{i, j}(t)\right]^{a 2}} ; w>w_{0}, n \in N_{i} \\
w_{0}=b_{1}+\frac{b_{2} I_{c}}{I_{\max }}
\end{array}\right.
$$

Where, $\zeta_{0}$ is initial pheromone intensity of each link between $N_{i}$ and $N_{j}, a_{1}$ and $a_{2}$ are the control parameters, $W$ is the random number uniformly distributed in $[0,1], W_{0}$ is the dynamic parameters that increases gradually with number of iterations (as Eq. (7)), $b_{1}$ and $b_{2}$ are constants, $I_{c}$ is the number of iterations and $I_{\max }$ is the maximum number of iterations.

$h_{t, j}(t)$ is the heuristic function i.e. the visibility of link between $N_{t}$ and $N_{i}$. It is defined using following Eq. (8).

$$
h_{i, j}(t)=\frac{1}{\left(\alpha Q_{j}+\delta d_{i, j}\right)^{\beta}}
$$

Where, $Q_{j}$ is the performance factor of $N_{i}$, $d_{i, j}$ is the distance, $\alpha$, and $\delta$ are proportional constants and $\beta$ is the loss constant.

4. Based on the estimated traffic arrival probability, the node traffic rate is adjusted with respect to the node distance. Simultaneously, each FANT finds its next hop node to gather the node data.

5. When FANT reaches its next hop node, the pheromone density $\varsigma$ of the link is updated based on the following local updating rule.

$$
\varsigma_{i, j}(t+1)=(1-y) \varsigma_{i, j}(t)+y \varsigma_{0}
$$

Where, $y(0<y<1)$ is the local pheromone decay parameter and $\varsigma_{0}$ is the initial value of pheromone.

6. The above steps 3, 4 and 5 are repeated until FANT establishes route set $G^{F A N T}$ 
7. When FANT obtains $G^{F A N T}$ among $S$ and $D$, the path evaluation function $F_{G}{ }^{F A N T}$ is estimated.

$$
\begin{aligned}
& F_{G} F A N T=\frac{\beta f_{D_{G} F A N T}+h f_{P L R}{ }_{G} F A N T}{V_{G} F A N T} \\
& V_{G^{F A N T}}=\rho \sum_{s \in S} \sum_{d \in D} E_{G_{s, d}^{F A N T}}+ \\
& \sum_{s \in S} \sum_{d \in D} Q L_{G_{s, d}^{F A N T}} ; \begin{array}{c}
F A N T \\
s, d
\end{array} G^{F A N T} \\
& f_{D_{G} F A N T}=\sum_{s \in S} \sum_{d \in D}\left[\omega _ { D } \left(D_{G_{s, d}^{F A N T}}-\right.\right. \\
& \left.\left.D_{c}\right)\right] ; G_{S, d}^{F A N T} \subset G^{F A N T} \\
& f_{P L R} G_{G A N T}=\sum_{s \in S} \sum_{d \in D}\left[\omega _ { p } \left(P L R_{G_{s, d}^{F A N T}}-\right.\right. \\
& \left.\left.P L R_{c}\right)\right] ; G_{s, d}^{F A N T} \subset G^{F A N T} \\
& \omega_{D}(M)=\left\{\begin{array}{c}
1 ; M \leq 0 \\
r_{d}\left(G_{s, d}^{F A N T}\right) ; \text { otherwise }
\end{array}\right.
\end{aligned}
$$

If delay value satisfies the delay constraint $D_{c}$, then the value of $\omega_{p}(M)$ is 1 , otherwise the value is $r_{d}\left(G_{s, d}{ }^{F A N T}\right)$.

$$
\omega_{p}(M)=\left\{\begin{array}{c}
1 ; M \leq 0 \\
r_{p}\left(G_{s, d}^{F A N T}\right) ; \text { otherwise }
\end{array}\right.
$$

If packet loss ratio value satisfies the constraint $P_{c}$, then the value of $\omega_{p}(M)$ is 1 , otherwise the value is $r_{d}\left(G_{s, d}{ }^{F A N T}\right)$.

$$
\begin{aligned}
& r_{d}\left(G_{s, d}^{F A N T}\right)=e^{-\left(D_{G_{s, d}^{F A N T}}-D_{c}\right)} \\
& r_{p}\left(G_{s, d}^{F A N T}\right)=e^{-\left(P_{\left.G_{S, d}^{F A N T}-P_{c}\right)}\right)}
\end{aligned}
$$

Where, $\beta, h, \rho, \tau$ are the positive weights of $f_{D G F A N T}, f_{P L R G}{ }^{F A N T}, \quad E_{G S d}{ }_{F A N T}$ and $Q L_{G s d}{ }^{F A N T}$ respectively. These values indecates the relative values of end-to-end delay, packet loss ratio, average energy consumption and queue length,

$$
V_{G^{F A N T}}=C
$$

Where $C$ is cost metrics, $\omega_{p}(M)$ is the metric function of delay and packet loss ratio parameter.

8. The performance factor of the node $P F_{i}$ with the queue length, forwarding packet number and residual energy is computed.

9. The maximum value of path evaluation function $F_{G}{ }^{F A N T}$ and global best route set $G_{\text {best }}^{\text {FANT }}$ is estimated.

$$
S_{i, j}(t+1)=(1-v) \varsigma_{i, j}(t)+v \triangle_{i, j}(t)
$$

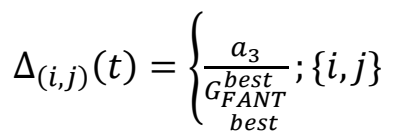

Where $v$ is global pheromone parameter, $a_{3}$ is constant, $G_{\text {FANTbest }}^{\text {best }}$ is global best route set and $\Delta_{t, j}(t)$ is increment of the pheromone intensity of link $\{i, j\}$. According to the global pheromone density updating rule, the pheromone density of the link is updated.

10. If the termination condition $\left(I_{c}>\operatorname{Imax}\right)$ is satisfied, then the algorithm is terminated. Otherwise, the above process is repeated.

After estimating the $N D$ and $T I$ value, the transfer time $\left(T_{x}\right)$ of the node is computed using function $T_{t x}=F(N D, T I)$. If $N D$ and $T I$ is high, assign $T_{t x}$ to high, otherwise $T_{t x}$ is assigned to low. The node with lowest transfer time is chosen as relay node during data transmission.

\subsection{Neighbor discovery}

Let $S$ and $D$ represent the source and destination respectively. Let $N_{i}$ be the intermediate node. Let $R R E Q$ and $R R E P$ be the route request and route reply message respectively. When source wants to transmit the data packet to destination node, it initiates the construction of DNT. The process is explained the following steps.

1) $S$ broadcast the $R R E Q$ message to its intermediate nodes $\left(N_{i}\right)$.

$$
S \stackrel{R R E Q}{\longrightarrow} N_{i}
$$

2) $N_{i}$ upon receiving $R R E Q$ on its beam ' $q$ ' updates its routing table with the information that includes source ID, destination ID, previous hop node ID, node distance and residual energy. It appends its state to the node state field of $R R E Q$ message and analyzes the destination ID. If $N_{i} \neq D$

Then, $N_{i}$ rebroadcasts $R R E Q$ to neighboring nodes

Else

$$
N_{i} \stackrel{R R E P}{\longrightarrow} S
$$

End if

3) In case $N_{i}$ receives two or more $R R E Q$ with similar destination ID, then it considers first received message and discards the other message. 
4) When $D$ receives $R R E Q$ message, it appends its state to RREP and unicasts the reply message in the reverse path in the same beam ' $q$ ' towards $S$. $D$ performs this similar action for every $R R E Q$ it receives.

$$
D \stackrel{R R E P}{\longrightarrow} S
$$

5) $N_{i}$ upon receiving the RREP message appends its state to the message and also updates its DNT. Then it unicasts the RREP in the direction of $\mathrm{S}$ utilizing the previous hop node information which is priorly stored.

6) Step 5 is repeated till RREP reaches $S$.

7) $S$ shares the updated DNT table with MAC layer.

8) Node overhears all the traffic in its vicinity once the MAC is set in promiscuous mode. i.e, the node can either updates it DNT or track new neighbors by overhearing the routing packets.

Once the neighbor nodes are discovered, $S$ uses ADMMAC protocol to transmit the data to the destination. This is explained in the next section.

\subsection{ADMMAC protocol}

In this protocol, multiple data are delivered to destination through a relay node equipped with a directional antenna following the Ready To Send (RTS)/ Clear To Send (CTS) exchange. The relay node selection is explained in section 3.4. Antenna model is explained in section 3.2.

We use the cooperative transmission mode when the node can transmit a data packet faster than direct transmission mode.

The steps involved in this protocol are as follows:

\subsubsection{RTS transmission}

1 If a node has a transmission frame, it senses the channel in omni-directional mode.

2 Once the transmitter confirms about the channel is free, the node transmits RTS frame to the receiver through the relay node using a directional antenna. The RTS frame consists of information of both the time duration of the DATA frame and the predefined data-fragment number denoted as $F$ in Fig 1.

3 The node in addition, sets a CTS-wait timer so as to prepare for the CTS frame reception with beam-forming towards the receiver.

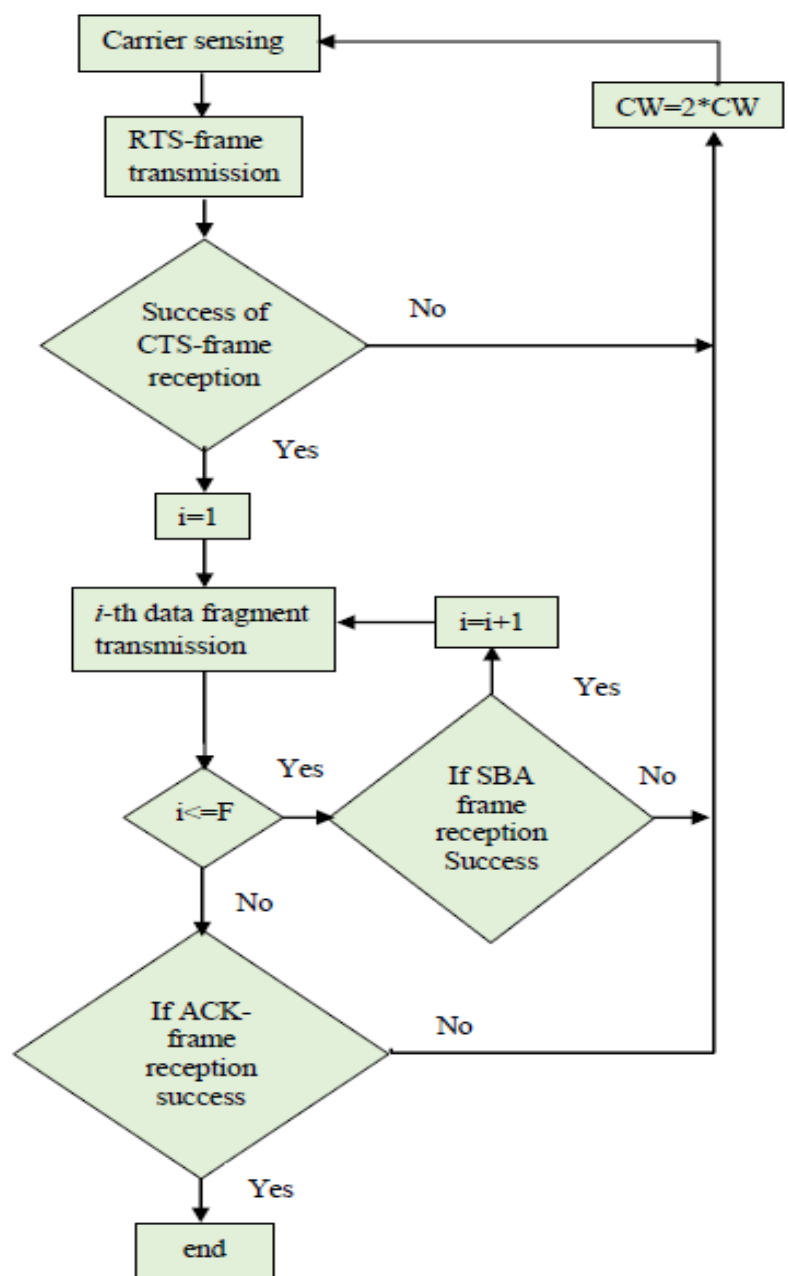

Figure.1 Flowchart for transmitter in ADMMAC protocol

4 If the transmitter could not receive CTS frame within the CTS-wait duration, it retransmits the RTS frame by doubling the Contention Window (CW) value.

5 If the transmitter receives CTS frame successfully, the transmitter starts the first datafragment transmission to the receiver through the relay node with directional antenna.

\subsubsection{CTS transmission}

1 Once a node receives RTS frame, the receiver transmits CTS frame to the transmitter with the time duration of the DATA frame and the datafragment number.

2 The data-fragment-length information is required to advertise the directional hidden node of the transmitter. Data-fragment length is calculated from the DATA-frame length and the data-fragment number included in the RTS frame.

3 Once the RTS/CTS is exchanged, the transmitter split the payload of the DATA frame into several fragments and inserts a small interval 
between two adjacent data-fragments. The interval is called Inter-Data-Frame Spacing (IDFS).

4 The receiver requires IDFS durations which include $T_{R T}$ and $T_{T R}$ to transmit Short Busy Advertise (SBA) signal. Where $T_{R T}$ and $T_{T R}$ denotes the time taken to switch from reception mode to transmission one and vice versa respectively.

5 On successful reception of the first data segment, receiver node sends the SBA signal to the neighbour nodes to inform the reception state of the receiver during the IDFS period.

6 SBA duration is long enough so that other nodes can sense the SBA signal. Any node sensing the SBA signal should keep silent for a certain period, namely "Inter-Frame Spacing due to Short Busy advertisement (BIFS)" by setting the Network Allocation Vector (NAV).

Note: Larger the data fragments, more number of IDFS should be included between two adjacent data-fragment transmissions.

7 The data fragment length is estimated based on ND and TI information estimated from section 3.4 using following Eq. (24).

$$
D F M=k_{\varsigma} G_{i, j}^{F A N T}(t)
$$

Where, DFM is data fragment length, $k$ is a constant, $\varsigma$ is a ND and $G_{i, j}{ }^{F A N T}(t)$ is TI information

8 The transmitter transmits the data fragments continuously with IDFS intervals.

9 If the receiver receives a data fragment successfully, the receiver sends the SBA signal for notifying not only the success of fragment reception to the transmitter but also the ongoing communication situation to directional-hidden nodes.

10 Once the receiver receives the last data fragment, it replies an Acknowledgement (ACK) frame to the transmitter through the relay nodes.

\subsubsection{Transmission verification and retransmission}

\subsubsection{Verification using SBA signal}

In the ADMMAC protocol, after every datafragment transmissions the transmitter checks the SBA signal from the receiver. If the transmitter could not detect the SBA signal successfully, it stops data-fragment-transmission process to avoid the unnecessary transmission-time wastage.

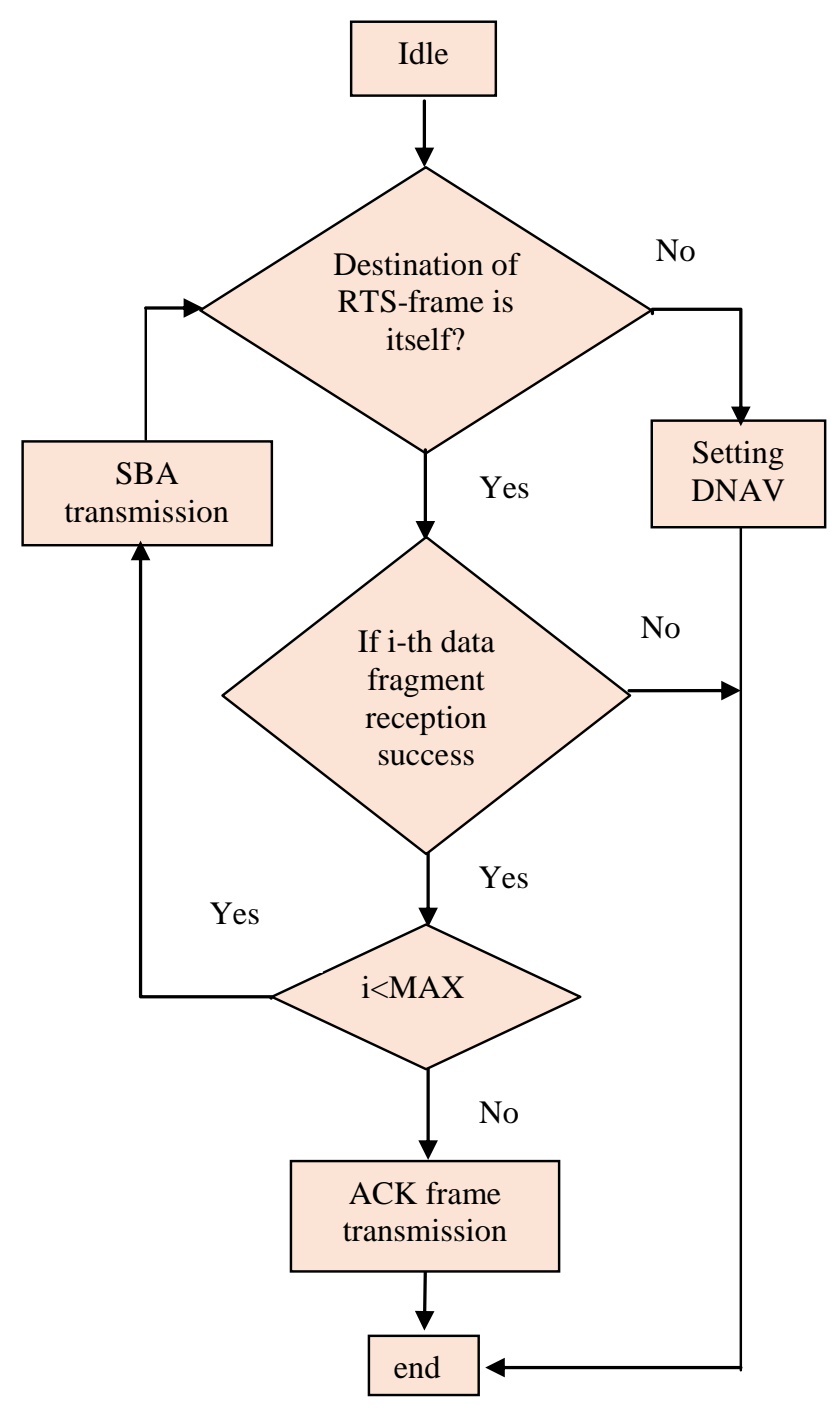

Figure.2 Flowchart for receiver in ADMMAC protocol

\subsubsection{Verification using ACK-wait timer}

When the transmitter completes a last datafragment transmission, the transmitter sets an ACKwait timer to wait for an ACK frame as a response. If the transmitter could not receive an ACK frame in the ACK-wait duration, it retransmits RTS frame by doubling the $\mathrm{CW}$. When the transmitter can receive the ACK frame, the transmitter recognizes that the frame transmission is succeeded.

\section{Simulation results}

\subsection{Simulation parameters}

We use NS2 to simulate our proposed CDRP. We use the IEEE 802.11 for wireless MAC layer protocol. It has the functionality to notify the network layer about link breakage. In our simulation, the packet sending rate is varied as 50, 100, 150, 200 and $250 \mathrm{~Kb}$. The area size is 1300 meter $x 1300$ 
meter square region for 50 seconds simulation time. The simulated traffic is Constant Bit Rate (CBR).

\subsection{Results and analysis}

We evaluate performance of the new protocol mainly according to the following parameters. Performance metrics of our proposed work CDRP are compared with the DRP [9] protocol and MAREERP [3]. The simulation results are presented in following sub-sections. Our simulation settings and parameters are summarized in table 1.

Table 1. Simulation parameters

\begin{tabular}{|l|l|}
\hline No. of Nodes & $20,40,60,80$ and 100 \\
\hline Area & 1300 X 1300 sq. meters \\
\hline MAC & 802.11 \\
\hline Simulation Time & $50 \mathrm{Sec}$ \\
\hline Traffic Source & CBR \\
\hline Rate & $50,100,150,200$ and $250 \mathrm{~Kb}$ \\
\hline Propagation & TwoRayGround \\
\hline Antenna & OmniAntenna \\
\hline
\end{tabular}

\subsubsection{Case-1 (Grid)}

In our first experiment we are varying the rate as 50, 100, 150, 200 and $250 \mathrm{~Kb}$ for CBR traffic. Figs. 3 to 6 show the results of delay, delivery ratio, packet drop and throughput by varying the rate from $50 \mathrm{~Kb}$ to $250 \mathrm{~Kb}$ for the CBR traffic in CDRP, MAREERP and DRP protocols. When comparing the performance of three protocols, we infer that CDRP outperforms MAREERP and CRP by 41$54 \%$ in terms of delay, $82-78 \%$ in terms of delivery ratio, $51-66 \%$ in terms of packet drop and $82-87 \%$ in terms of throughput.

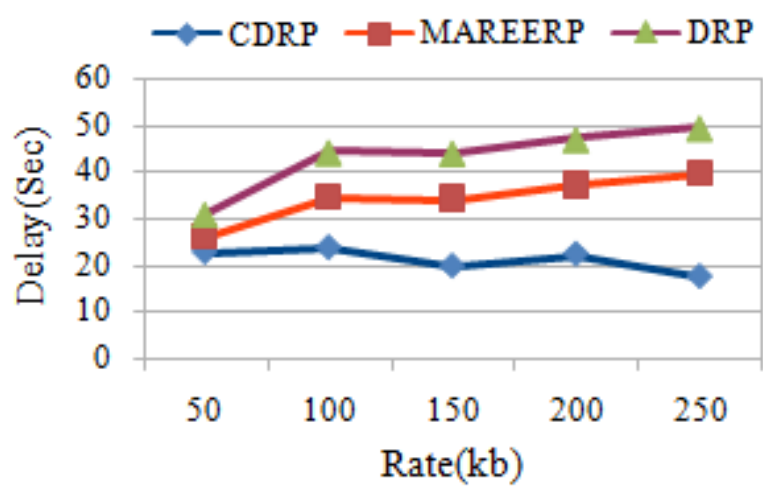

Figure.3 Rate Vs Delay

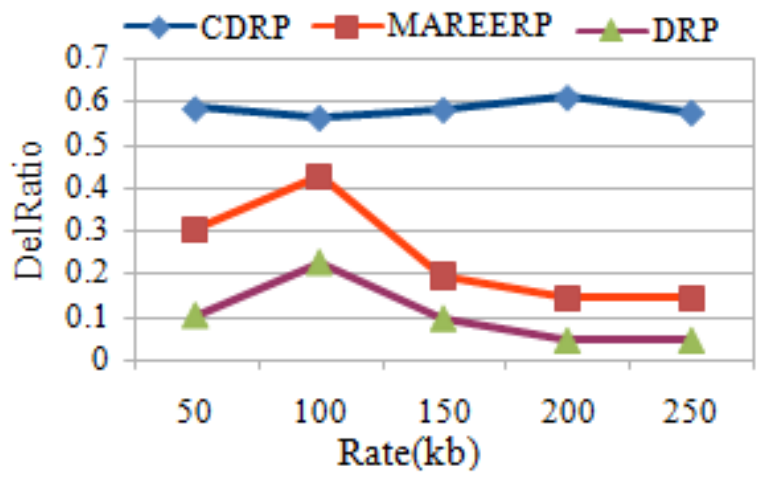

Figure.4 Rate Vs DeliveryRatio

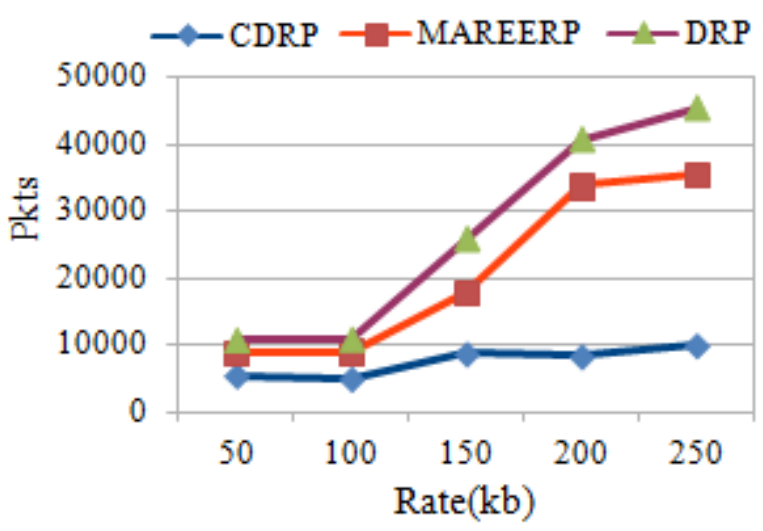

Figure.5 Rate Vs Drop

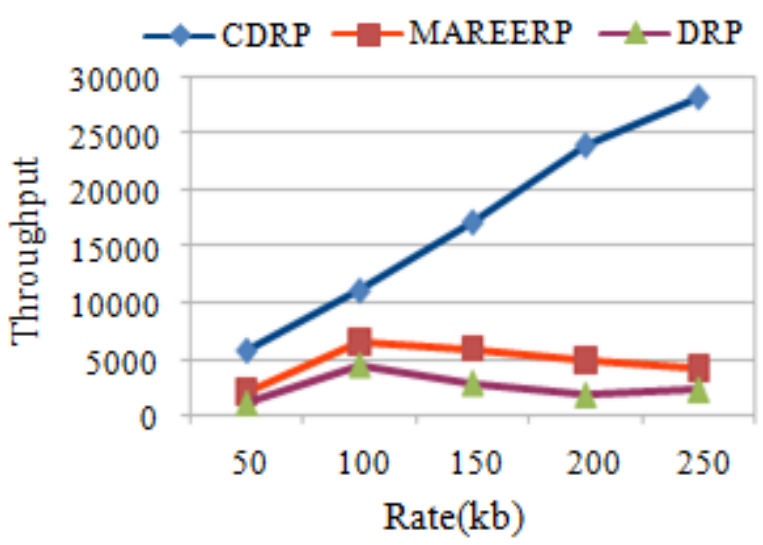

Figure.6 Rate Vs Throughput

\subsubsection{Case-2 (Non-Linear)}

\subsubsection{Based on nodes}

In our second experiment we vary the number of nodes as 20,40,60,80 and 100. Figs. 7 to 9 show the results of delay, delivery ratio and throughput by varying the nodes from 20 to 100 for the CBR traffic in CDRP, MAREERP and DRP protocols. When comparing the performance of three protocols, we infer that CDRP outperforms MAREERP and DRP by $40-55 \%$ in terms of delay, $26-46 \%$ in terms of delivery ratio and $50-68 \%$ in terms of throughput. 


\subsubsection{Based on Rate}

In our second experiment we vary the transmission rate as $50,100,150,200$ and $250 \mathrm{~Kb}$. Figs. 10 to 12 show the results of delay, delivery ratio and throughput by varying the rate from $50 \mathrm{~Kb}$ to $250 \mathrm{~Kb}$ for the CBR traffic in CDRP, MAREERP and DRP protocols. When comparing the performance of three protocols, we infer that CDRP outperforms MAREERP and CRP by $42-56 \%$ in terms of delay, $14-28 \%$ in terms of delivery ratio and $48-62 \%$ in terms of throughput.

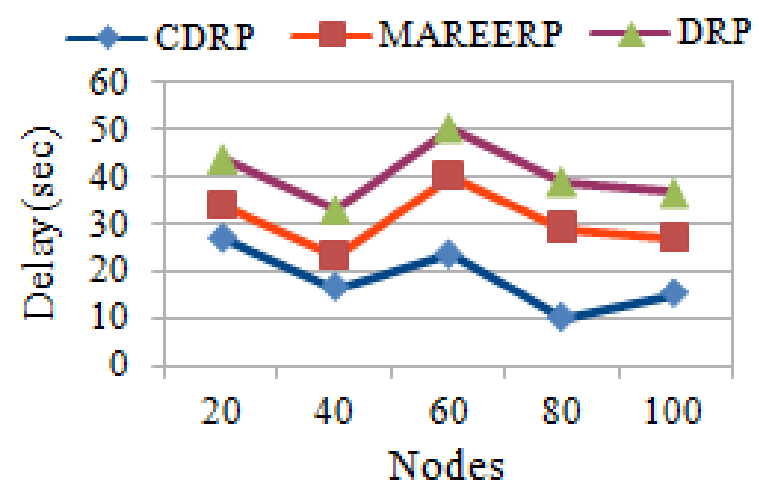

Figure.7 Nodes Vs Delay

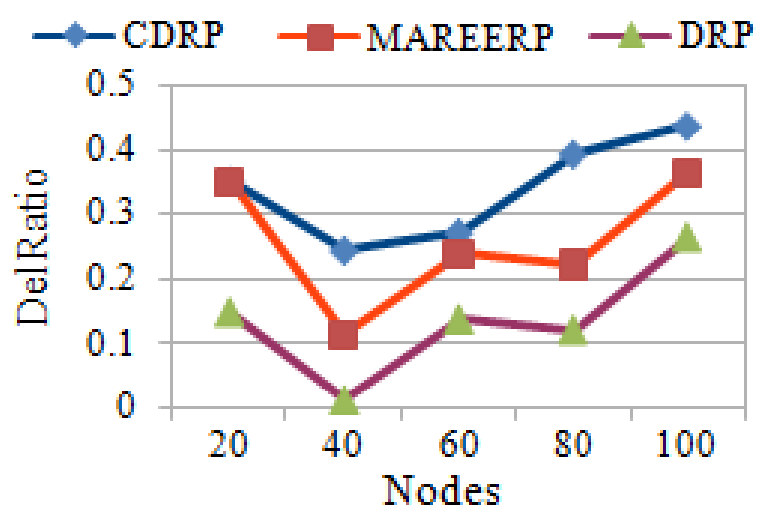

Figure.8 Nodes Vs Delivery Ratio

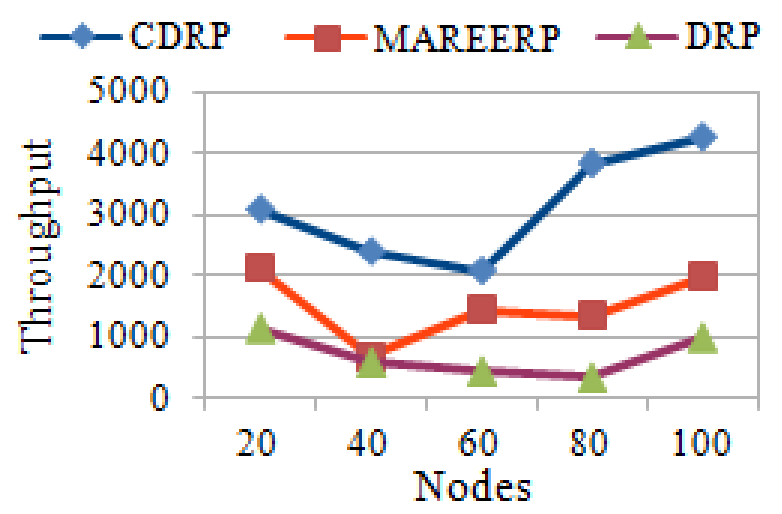

Figure 9. Nodes Vs Throughput

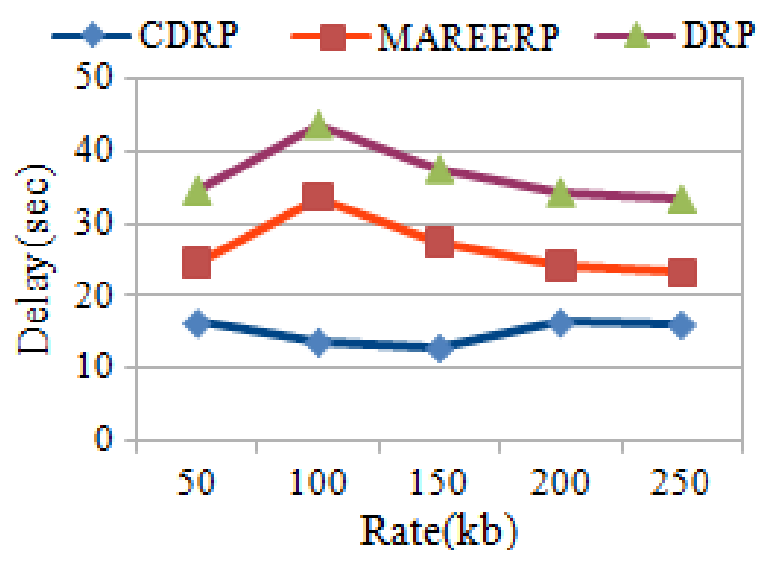

Figure.10 Rate Vs Delay

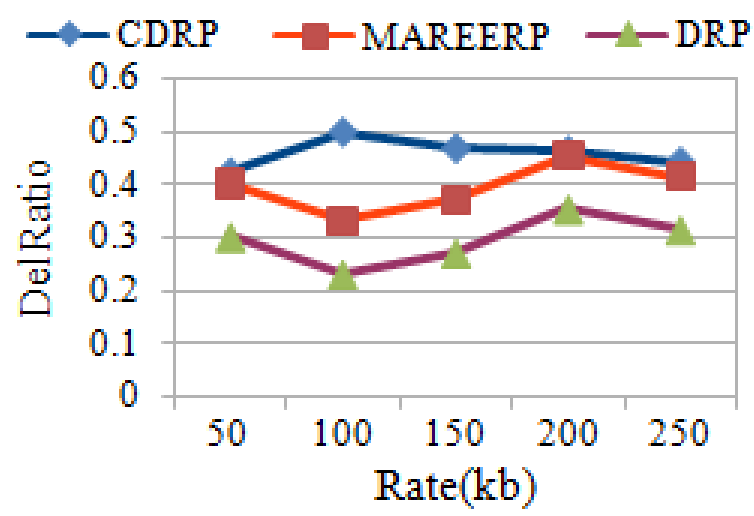

Figure.11 Rate Vs Delivery Ratio

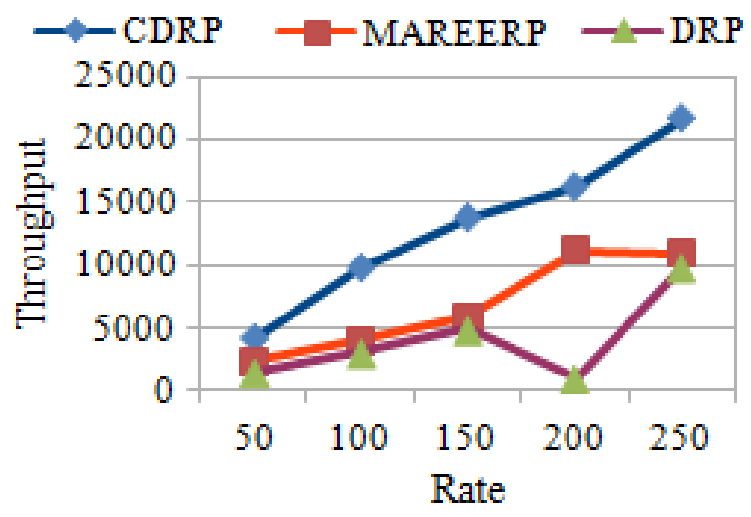

Figure.12 Rate Vs Throughput

\section{Conclusion}

In this paper, we have proposed to design a CDRP in MANET. Initially the source nodes discover neighbors by constructing the DNT. Then the node with minimum transfer time is selected as best relay node. The transfer time is estimated from ND and TI information collected by ant agents. Once the relay nodes are selected, the source node follows the ADMMAC protocol to transmit the data to the destination. By simulation results, we have shown that the proposed technique minimizes the drop by $51-66 \%$ and enhances the throughput by 48 - 
$62 \%$. As a future work, energy efficiency of the network will be improved using the optimized directional routing protocol.

\section{References}

[1] M. A. Mikki, "Energy Efficient Location Aided Routing Protocol for Wireless MANETs", International Journal of Computer Science and Information Security (IJCSIS), Vol.4, No.1 \& 2, 2009.

[2] P. B. Jarande and M. M. Patil, "Restricted Flooding and Directional Routing for Wireless Mobile Adhoc Network", International Journal of Computer Applications (IJCA), In: Proc. on Emerging Trends in Electronics and Telecommunication Engineering 2013, pp. 2530, 2014.

[3] S. Nallusamy, S. Appavupillai, and S. Ponnusamy, "Mobile Agents based Reliable and Energy Efficient Routing Protocol for MANET", International Journal of Intelligent Engineering and Systems, Vol.9, No.3, pp.110-116, 2016.

[4] H. Gossain, T. Joshi, C. Cordeiro, and D. P. Agrawal, "DRP: An Efficient Directional Routing Protocol for Mobile Ad Hoc Networks", IEEE Computer Society, Vol.17, No.12, pp.1438-1541, 2006.

[5] L. A. Latiff, N. Fisal, S. A. Arifin, and A. Ali Ahmed, "Directional Routing Protocol in Wireless Mobile Ad Hoc Network", Trends in Telecommunications Technologies, Christos J Bouras (Ed.), 2010.

[6] L. A. Latiff, A. Ali, C. C. Ooi, N. Fisal, and M. Ismail, "Network Performance of a Multi-hop Quadrant based Directional Routing Protocol (Q-DIR) in Wireless Mobile Ad Hoc Network", In: Proc. of International Conf. on Computing \& Informatics, pp.1-5, 2006.

[7] J. Shen, W. Zheng, J. Wang, Z. Xia, and Z. Fu, "Routing Protocols using Directional Antennas in Ad Hoc Networks: A Comparative Review", International Journal of Grid and Distributed Computing, Vol.6, No.5, pp.39-50, 2013.

[8] H. Gossain, T. Joshi, C. Cordeiro, and D. P. Agrawal, "A Cross-Layer Approach for Designing Directional Routing Protocol in MANETS", In: Proc. of Wireless Communications and Networking, Vol.4, pp.1976-1981, 2005.

[9] V. Kolar, P. Rogers, and N. B. Abu-Ghazaleh, "Route Compaction for Directional Route Discovery in MANETs", In: Proc. of International Conf. on Wireless and Mobile
Computing, Networking and Communication, Vol.3, pp.101-108, 2005.

[10] S. Chaudhari and R. Biradar, "Traffic and mobility aware resource prediction using cognitive agent in mobile ad hoc networks", Journal of Network and Computer Applications, Vol.72, pp. 87-103, 2016.

[11] S. González, W. Castellanos, P. Guzmán, P. Arce, and J. Guerri, "Simulation and experimental testbed for adaptive video streaming in ad hoc networks", Ad Hoc Networks, Vol. 52, pp. 89-105, 2016.

[12] C. Lal, V. Laxmi, M. Gaur, and M. Conti, "Enhancing QoE for video streaming in MANETs via multi-constraint routing", Wireless Network, Vol. 24, No. 1, pp. 235-256, 2018.

[13] Y. Bao, W. Lei, W. Zhang, and Y. Zhan, "QoE collaborative evaluation method based on fuzzy clustering heuristic algorithm", Springer Plus, Vol.5, No.1, pp.1-26, 2016.

[14] N. Boddu, R. Vatambeti, and V. Bobba, "Achieving Energy Efficiency and Increasing the Network Life Time in MANET through Fault Tolerant Multi-Path Routing", International Journal of Intelligent Engineering and Systems, Vol.10, No.3, pp.166-172, 2017.

[15] B. Rajkumar and G. Narsimha, "Secure Light Weight Encryption Protocol for MANET", International Journal of Intelligent Engineering and Systems, Vol.10, No.3, pp.58-65, 2017. 